\title{
Early well differentiated thyroid cancer: is total thyroidectomy still indicated?
}

\author{
Antonio E Alfonso* \\ Professor of Surgery, Zucker School of Medicine at Hofstra/Northwell
}

The topic of total thyroidectomy (TT) in early well differentiated papillary thyroid cancer has always continued to provide ample fodder for discussion. The extent of resection (total thyroidectomy $v s$. lobectomy/isthmusectomy) in its application to "early papillary thyroid cancer" continue to be debated and, sometimes, rather passionately.

Statistical advantages utilizing long term survival or cancer mortality between both procedures are comparable and are no longer debated issues provided the occasional modest but recognized recurrences after conservative resection, in either the residual lobe or in lymph nodes, are both diagnosed and surgically addressed in a timely fashion.

However, in those who had less than TT resections, lifelong monitoring of the residual lobe and further additional serial studies, i.e., sonography, will be necessary and can become both tedious and ultimately more costly. Furthermore, in those who will require reoperative surgery, the subsequent operation can be even technically more challenging, especially when the previous surgery may have involved manipulation of the residual contra lateral lobe and para tracheal area, and be accompanied by a higher complication rate.

On the other hand, the immediate complications of possible permanent hypoparathyroidism and of bilateral nerve injury remain the key elements of concern in the choice of determining whether TT performed over a lesser resection in such earlier stage thyroid cancers.

Total thyroidectomy is in reality a parathyroid preservation operation and while a routine meticulous dissection in the hands of experienced and usually high volume surgeons can avoid hypoparathyroidism, most of thyroid operations throughout most communities in the United States are actually currently performed by board certified general surgeons or otolaryngologists who, in fact, do not routinely do a large volume of thyroid operations.

The complications of thyroid surgery are directly proportional to the extent of the thyroidectomy and inversely to the experience of the operating surgeon [1] and it is the individual surgeons' own honestly assessed immediate complication rate that should become the key consideration in the choice between TT and a lesser resection when applied to early well differentiated papillary thyroid cancer.

The issue with any recommended guidelines is that they should be applicable to all surgeons and not just "experts" and this may well be a key consideration for the recent modifications in the 2016 ATA guidelines from their former 2009 recommendations.

Copyright: (C2017 Alfonso AE. This is an open-access article distributed under the terms of the Creative Commons Attribution License, which permits unrestricted use, distribution, and reproduction in any medium, provided the original author and source are credited.
Furthermore, with the increasing use of diagnostic neck sonography, there has been a noticeable increase not only in the incidence of thyroid papillary cancers diagnosed at much earlier stages, but more so, in the proportionate increase in papillary thyroid microcarcinomas [PTMC].

More recently, Pitt and colleagues have demonstrated not only a high incidence of multifocality, lymph node metastases and recurrences in PTMC but also noted a distinctly higher incidence of bilaterality (53\%) when the PTMC was multifocal in the index lobe [2].

Now while most PTMC are not biologically aggressive, some are. Noguchi et al observed a quadruple increase in recurrence incidence when the micro cancer was between $5 \mathrm{~mm}$. to $10 \mathrm{~mm}$. in size versus those less than $5 \mathrm{~mm}$. Aside from increasing size, other independent prognostic risk factors identified were capsular invasion, extra thyroidal tumor extension and, of course lymph node metastases [3].

He and colleagues have reinforced size and added multifocality, bilaterality and age ( $>45)$ as all independent risk factors [4].

Our own study demonstrated that when PTMC was multifocal in a subset of patients over 45 years of age, the incidence of bilateral disease increased to $76 \%$ [5]. Total thyroidectomy, when it can be accomplished safely in experienced hands, may be an option well worth considering not only in this situation, but also in the not infrequent circumstance when multifocal PTMC is incidentally demonstrated on paraffin sections after a lobectomy has been completed for a multinodular goiter in a patient over 45 years of age.

Total thyroidectomy, done well and in experienced hands, may still be worth performing in early well differentiated papillary thyroid cancers.

\section{References}

1. Sosa J, Bowman H, Tielsch J, Powe N (1998) The importance of Surgeon Experience for Clinical and economic outcomes from thyroidectomy. Ann Surg 226: 320-330 [Crossref]

2. Pitts C, Sippel R.S, Chen H (2009) Contralateral papillary thyroid cancer: does size matter. Am J Surg 197: 342-347 [Crossref]

3. Noguchi S, Yamnshita H, Uchino S, Watanake S (2008) World J surgery 32: 747-753

4. He Q, Zhuang D, Zheng L, Fan Z (2012) The Surgical management of papillary thyroid microcarcinoma: a 162 month single-center experience of 273 cases. Amer Surgery 78 : 1215-1218 [Crossref]

5. Ricci J, Alfonso A (2012) Multifocal micropapillary thyroid cancer: A new indication for total thyroidectomy. Amer Surg 78: 1211-1214 [Crossref]

Correspondence to: Antonio E Alfonso, MD, FACS, Professor of Surgery, Zucker School of Medicine at Hofstra/Northwell, Vice Chairman of Surgery \& Director of Surgical Education, Northwell Health/USA, E-mail: aalfonso1@northwell.edu

Received: November 03, 2017; Accepted: November 20, 2017; Published: November 24, 2017 